\title{
Phase-Change Metadevices for the Dynamic and Reconfigurable Control of Light
}

\author{
C D Wright ${ }^{1}$, S G-C Carrillo ${ }^{1}$, C R de Galarreta ${ }^{1}$, E Gemo ${ }^{1}$, L Trimby ${ }^{1}$, A M Alexeev ${ }^{1}$, Y-Y Au ${ }^{1}$, V K \\ Nagareddy $^{1}$, A Baldycheva ${ }^{1}$, J Bertolotti ${ }^{1}$, M Lopez-Garcia ${ }^{2+}$, M Klemm ${ }^{2}$, M Cryan ${ }^{2}$ \\ College of Engineering, Mathematics and Physical Sciences, University of Exeter, EX4 4QF, UK \\ ${ }^{2}$ Department of Electrical and Electronic Engineering, University of Bristol, Bristol, BS8 1TH, UK. \\ +Now at: Nanophotonics Dept., International Iberian Nanotechnology Laboratory (INL), 4715-330, Braga, Portugal
}

\begin{abstract}
The combination of chalcogenide phase-change materials with optical metamaterial arrays is exploited to create new forms of dynamic, tuneable and reconfigurable photonic devices including perfect absorbers, modulators, beam steerers and filters. () 2018 The Author(s)
\end{abstract}

\section{Introduction}

Chalcogenide phase-change materials, such as the well-known alloy $\mathrm{Ge}_{2} \mathrm{Sb}_{2} \mathrm{Te}_{5}$, have been widely researched and exploited for the provision of non-volatile optical and electrical memories [1]. Their utility in such applications arises due the large differences in their optical and electrical properties when in amorphous and crystalline phases, and the ability to switch between these phases quickly and repeatedly via electro-thermal or opto-thermal excitation. More recently, the use of chalcogenide phase-change materials as a form of switchable/tuneable dielectric in optical metamaterial structures, such as metal-dielectric-metal plasmonic resonators and all-dielectric metamaterials, has generated much interest [2]. In this context, the ability to control the refractive index and absorption ( $\mathrm{n}$ and $\mathrm{k}$ ) of the phase-change material by controlling (via suitable in-situ or ex-situ excitation) its degree of crystallisation/amorphisation allows us to realise a whole new generation of dynamic, tuneable and reconfigurable optical metadevices. Here we demonstrate just some of these possibilities, by showcasing phase-change metadevices that can act as switchable absorbers, optical beam-steerers, NIR light modulators, tuneable MIR filters and metasurface-based phase-change optoelectronic displays [3].

\section{Device design and operation}

By way of example, Figure 1 shows two typical phase-change metadevice structures that we have explored. The first, shown in Figure 1(a), can operate as both a 'perfect' metamaterial absorber and as an optical modulator [4]. It has a patterned top plasmonic metal layer, an ITO layer, a phase-change layer (here shown as $\mathrm{Ge}_{2} \mathrm{Sb}_{2} \mathrm{Te}_{5}$, or GST for short) and a continuous (i.e. un-patterned) bottom metal layer. The ITO layer provides environmental protection for the GST layer (which oxidises easily if exposed to air), while also allowing for both optical and electrical access to the device. The device in Figure 1(b) is slightly more complex, in so much as the top patterned metasurface has metal strips of differing widths, and the dielectric space is filled with both GST and $\mathrm{SiO}_{2}$; this device can provide a dynamic beam-steering capability [5]. The simulated and experimental performance of both devices is shown in Figure 1(c) and (d), for the case of an operating wavelength of 1550 $\mathrm{nm}$. It is clear the device of Figure 1(a) acts as an absorber when the GST is in the amorphous state, and as a reflector when it is in the crystalline state; switching between states, e.g. by electro-thermal switching of the GST layer, thus leads to a modulating capability and demonstrates a high modulation index. The device of Figure 1(b) reflects a normally incident plane wave in a mirror-like fashion when the GST layer is crystalline, but reflects in an anomalous fashion at a pre-designed angle (here $\sim 32^{\circ}$ ) when the GST is amorphous. The beam-steering efficiency is around $40 \%$, a very high value for plasmonic-type devices.

The devices of Figure 1 both operate in reflection and use plasmonic and Fabry-Perot resonances to control and tailor the optical performance. In Figure 2(a) we show an approach that works in transmission, here exploiting so-called extraordinary optical transmission (arising from a periodic array of circular holes in a thin metal film) in combination with phase-change materials, to deliver a dynamically tuneable and reconfigurable mid-infrared filter. The geometry of the device is designed to transmit light at a specific wavelength (here $\sim 3.3$ $\mu \mathrm{m}$ ) when the phase-change layer (here again GST) is in the amorphous phase, but when the GST is switched to the crystalline state the centre frequency of the transmission shifts, as shown in Figure 2(b). Indeed, by controlling the fraction of crystallisation in the GST layer, a single filter with multiple pass bands (and high efficiency) can be achieved, potentially finding application in areas such as multi-spectral imaging. 
Finally, to improve efficiencies beyond those achieved by typical plasmonic-type device structures, we have also explored the use of dielectric metamaterial approaches, such as that shown in Figure 3. Here we use a hybrid dielectric/metal plasmonic resonator, with a bottom metal (Au) plane and a top hybrid silicon/GST cubic resonator. Such devices can deliver, for example, beam steering with very high efficiencies (over $65 \%$ for the type of structure shown in Figure 3).
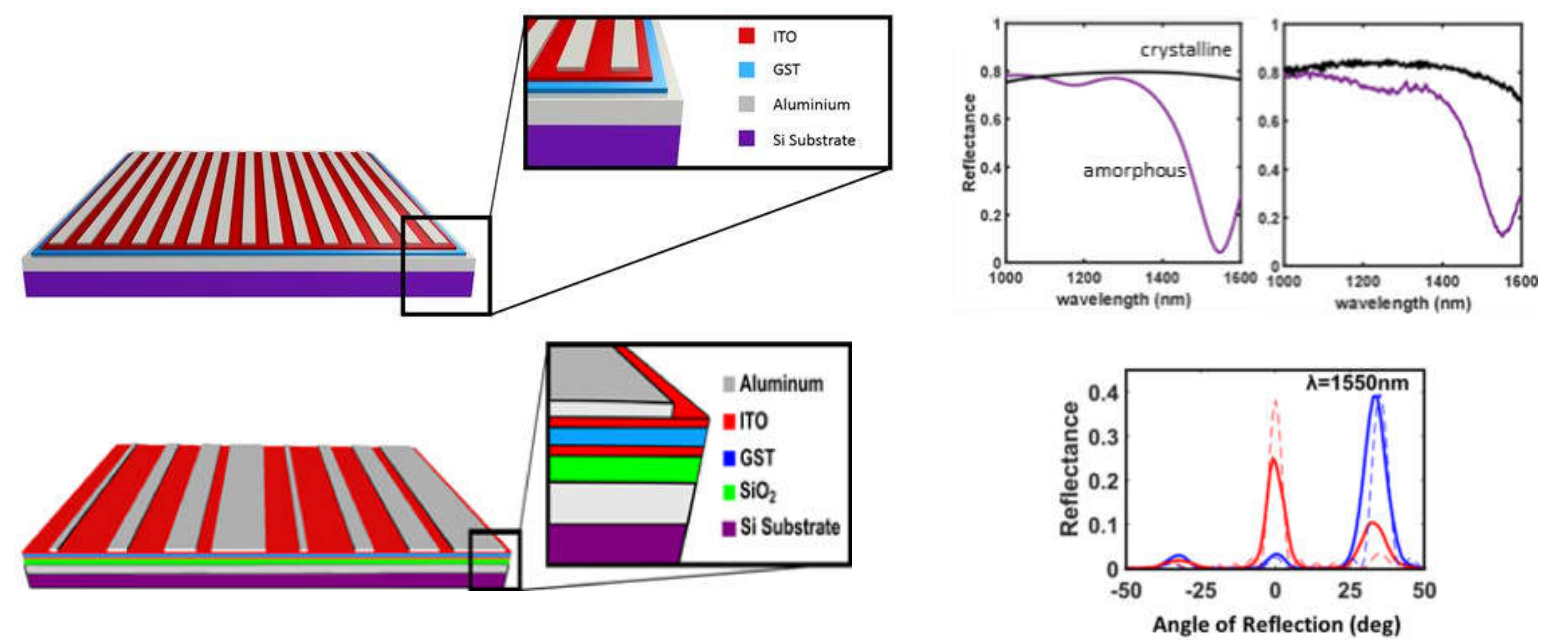

Figure 1: (a) (top left) Typical structure of a phase-change meta-absorber/modulator type device and (b) (top right) simulated (left) and (right) experimental reflectance spectra. (c) (bottom left) A plasmonic phase-change beam steering device structure and (d) (bottom right) simulated (dotted lines) and experimental (solid lines) angular reflectance with the GST layer crystalline (red) and amorphous (blue).
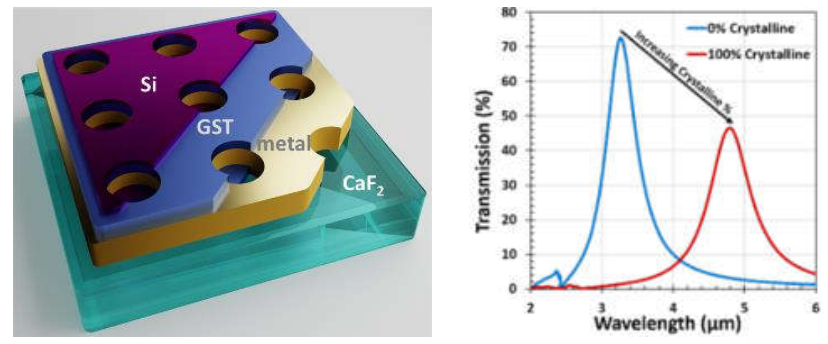

Figure 2: (a) (left) Schematic of a dynamically tuneable bandpass phase-change meta-filter and (b) (right) simulated response of such a filter with GST layer in fully amorphous and fully crystalline states.

\section{Summary}

We have shown that appropriately designed combinations of phase-change materials and metamaterial arrays opens-up a route to a world of new photonics functionalities.

\section{Acknowledgments}

CDW and VKN acknowledge ONRG funding (\#N62909-16-1-2174). CDW, AMA, Y-YA, VKN acknowledge EPSRC funding EP/M015130/1 \& EP/M015173/1. CrdeG, SG-CC, EG and LT the EPSRC CDT in Metamaterials (EP/L015331/1). LT acknowledges support from QinetiQ. MLG acknowledges EPSRC funding EP/M009033/1.

\section{References}

[1] M Wuttig and N Yamada, Nature Materials 6, 824 (2007)

[2] M Wuttig, H Bhaskaran and T Taubner, Nature Photonics 11, 465 (2017)

[3] P Hosseini, C D Wright and H Bhaskaran, Nature 511, 206 (2014)

[4] S G-C Carrillo et al., Optics Express 24, 13563 (2016)

[5] C Ruiz de Galarreta et al., Advanced Functional Materials, doi:10.1002/adfm.20170499 (2018)
Figure 3: (a) (top) A hybrid dielectric-plasmonic phase-change beam-steering device and (b) (bottom) simulated performance with the GST layer in amorphous (left) and crystalline (right) states (efficiency in both states $\sim 65 \%$ ). 